International Journal of Bifurcation and Chaos, Vol. 16, No. 3 (2006) 693-700

(c) World Scientific Publishing Company

\title{
GENERAL CONSIDERATION FOR MODELING AND BIFURCATION ANALYSIS OF SWITCHED DYNAMICAL SYSTEMS
}

\author{
YUE MA* and HIROSHI KAWAKAMI ${ }^{\dagger}$ \\ Department of Electrical and Electronic Engineering, \\ University of Tokushima, Tokushima 770-8506, Japan \\ *mayue@ee.tokushima-u.ac.jp \\ †kawakami@ee.tokushima-u.ac.jp \\ CHI K. TSE \\ Department of Electronic and Information Engineering, \\ Hong Kong Polytechnic University, Hunghom, Hong Kong \\ encktse@polyu.edu.hk \\ TAKUJI KOUSAKA \\ Department of Electronic and Electrical Engineering, \\ Fukuyama University, Japan \\ takuji@fuee.fukuyama-u.ac.jp
}

Received January 31, 2005; Revised February 25, 2005

\begin{abstract}
In much of the previous study of switched dynamical systems, it has been assumed that switching occurs at a common border between two regions in the same space as the system trajectory crosses the border. However, models arising from this consideration cannot cover systems whose trajectories do not actually "cross" the border. A typical example is the current-mode controlled boost converter whose trajectory is "reflected" at the border. In this paper, we propose a general method to model switched dynamical systems. Also, we suggest an analytical procedure to determine periodic solutions and their stability. The method is developed in terms of solution flows, and no solution has to be explicitly written. Most practical switched dynamical systems can be modeled and analyzed by this method.
\end{abstract}

Keywords: Switched dynamical systems; modeling; bifurcation analysis; switching converters.

\section{Introduction}

Bifurcations arising from switched dynamical systems are important phenomena not only in theoretical context but also in practice. Many practical systems, such as switching power converters, chaos generators, variable structure controllers, etc., can be modeled as switched dynamical systems [Banerjee \& Verghese, 2001], and the analysis of bifurcations in these systems has been found very useful in determining the possible operating regimes as well as in locating the relevant operating boundaries [Tse, 2003].

Up to now, models for studying switched dynamical systems have focused on "switchings" which have been triggered by the crossing of the system trajectory over some operating borders in the state space [Kousaka et al., 1999; Ma et al., 2004]. Essentially, one or more predefined common borders divide the state space into two or more separate regions. The dynamics in different regions are 

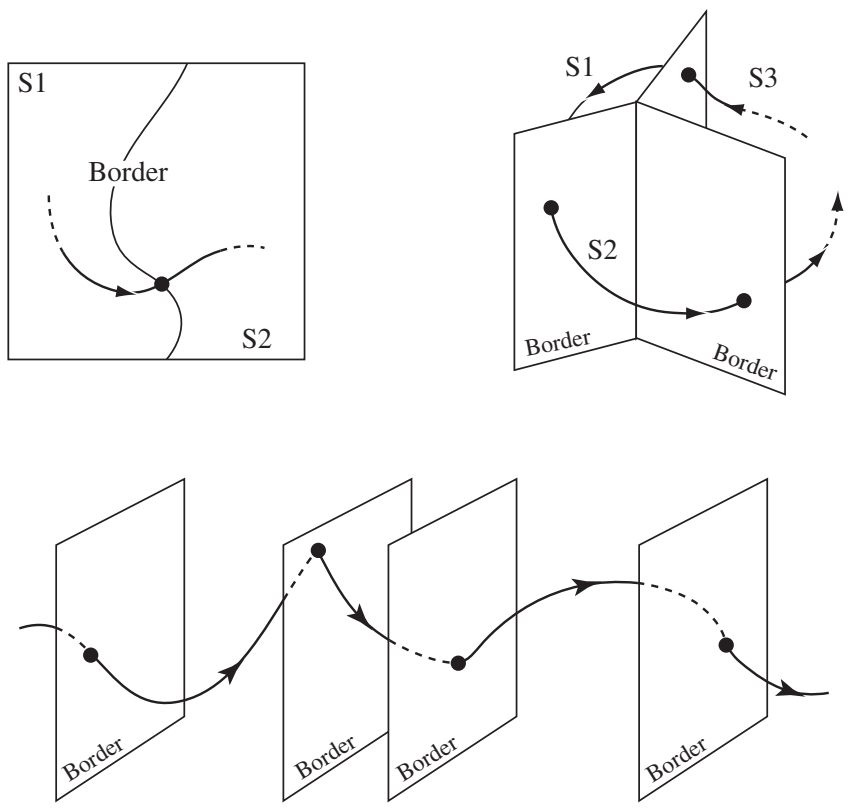

Fig. 1. Present models of switched dynamical systems.

governed by different system equations. As the trajectory crosses a border and moves into another region, switching occurs and the system is redefined [Banerjee et al., 2001; Kousaka et al., 1999; Kousaka et al., 2001], as illustrated in Fig. 1.

Several questions arise from this modeling approach. Does the trajectory move in the same space all the time? Does it always move across the border as it hits the border? Is the border always invariable?

As an example, the operation of the currentmode controlled boost converter may shed some light on the aforementioned questions [Chan \& Tse, 1997]. Referring to Fig. 2(a), the switch is turned off when the inductor current $i_{L}$ is equal to a reference current $I_{\text {ref }}$. A clock signal turns on the switch periodically at $t=k T$. During the on-time, $i_{L}$ climbs to the value of $I_{\text {ref }}$, and then ramps down during the off-time. A typical waveform of the inductor current is shown in Fig. 2(b). Thus, we can see that (i) instead of crossing the "border" at the turn-off instant, the solution trajectory is being "reflected" there; (ii) one border is available for one state, i.e. the border is not common. This kind of switching scenarios in fact represent a large class of applications, which has been referred to as event-driven switching [Krupar et al., 2004]. In order to cover such systems in a general model, we need to revise the basic modeling assumptions. One of our purposes in this paper is to present a more general method for modeling switched dynamical

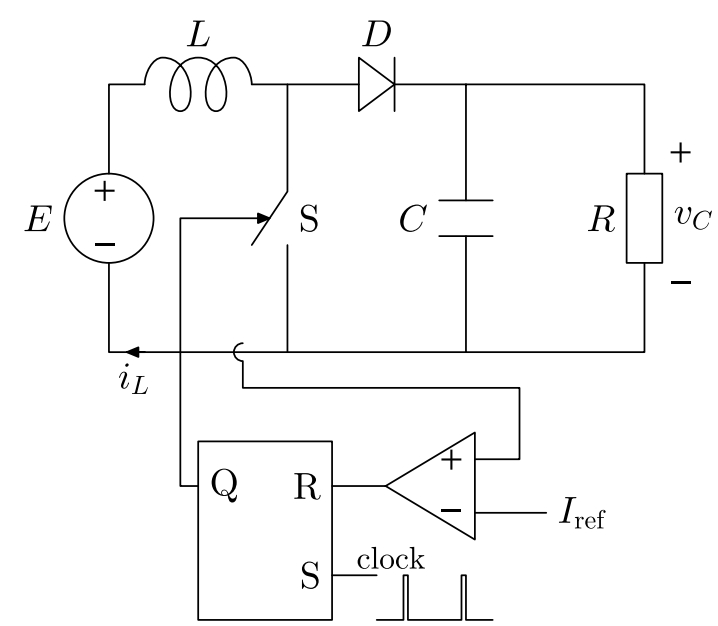

(a)

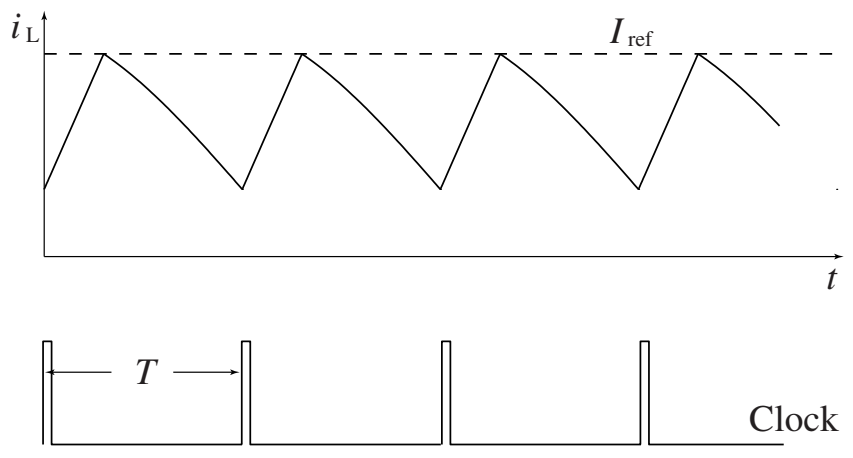

(b)

Fig. 2. (a) Current-mode controlled boost converter; (b) typical inductor current waveform.

systems that can cover most possible switching scenarios.

To analyze the dynamical behavior of a system, periodic solutions and their stability are often considered. By now, most studies have been performed in terms of piecewise linear ordinary differential equations, whose solutions are usually written as exponential functions. In this paper, however, we describe the solution of a switched system in terms of the flow in each space and propose a computational method which is independent of the form of the solutions. Our method can be extended to piecewise nonlinear systems.

\section{General Method of Modeling Switched Dynamical Systems}

As mentioned earlier, one consequence of the basic deficiencies in conventional modeling methods for switched dynamical systems is the failure 
in modeling switched systems where solutions do not actually cross the border(s), e.g. those operating under event-driven switchings such as the currentmode controlled switching converter. In this section, we begin with a reformulation of the operating features that cover a more general class of switched dynamical systems including those operating under event-driven switching. These features are valid for most switched dynamical systems, and include

1. Each subsystem moves in its own space. Spaces are isolated (not divided).

2. There is at least one border in each space. A solution can only move on one side of the border. Switching occurs if, and only if, the solution hits the border in its own space.

3. Switching action is not border crossing. Instead, it is more precisely described as "jumping between spaces".

Let us consider a switched dynamical system consisting of $m$ subsystems: $S_{1}, S_{2}, \ldots, S_{m}$.

$$
\begin{gathered}
S_{1}: \dot{x}=f_{1}\left(x, \lambda_{1}\right), \quad x \in R^{n}, \\
\vdots \\
S_{m}: \dot{x}=f_{m}\left(x, \lambda_{m}\right), \quad x \in R^{n},
\end{gathered}
$$

where $\lambda_{1,2, \ldots, m}$ are system parameters. There are $m$ spaces, namely, $M_{1}, M_{2}, \ldots, M_{m}$, corresponding to the $m$ subsystems. The borders in each space are

$$
\begin{gathered}
B_{1}=\left\{x \in R^{n}, t \in R: \beta_{1}(x, t)=0\right\} \\
\vdots \\
B_{m}=\left\{x \in R^{n}, t \in R: \beta_{m}(x, t)=0\right\}
\end{gathered}
$$

where $\beta_{1,2, \ldots, m}$ define the switching conditions. Note that $\beta_{i}(\cdot)=0$ may not be a single equation, since multiborders are possible for each space and solutions may jump to a different space at different borders. Thus, $M_{k}(k=1, \ldots, m)$ is the portion obtained by removing the part on one side of the "border" $B_{k}(k=1, \ldots, m)$,

$$
\begin{gathered}
M_{1}=\left\{(x, t) \in R^{n} \times R: \beta_{1}(x, t) \geq(\text { or } \leq) 0\right\} \\
\vdots \\
M_{m}=\left\{(x, t) \in R^{n} \times R: \beta_{m}(x, t) \geq(\text { or } \leq) 0\right\}
\end{gathered}
$$

The solution of the system in $M_{k}$ is governed by the state equations corresponding to $S_{k}$, as given in (1). Suppose the solutions in $M_{k}(k=1, \ldots, m)$ exist and are given by

$$
\begin{array}{ll}
x(t)=\varphi_{1}\left(t, x_{0}\right), & x(0)=x_{0}, \quad(x, t) \in M_{1} \\
& \vdots \\
x(t)=\varphi_{m}\left(t, x_{0}\right), & x(0)=x_{0}, \quad(x, t) \in M_{m}
\end{array}
$$

where $x_{0}$ is the initial point.
The flow jumps from one space to another when it hits the border. Note that, the jumps between spaces do not lead to jumps of the value of state variables. Thus, the point at which a flow hits the border can be thought of as the initial point of the successive flow in the next space. This formulation is illustrated in Fig. 3. We can thus draw its phase portrait on a projection plane.

Note that, if $\beta_{k}(\cdot)=\beta_{k+1}(\cdot)$, borders $B_{k}$ and $B_{k+1}$ are identical. In that case, $M_{k}$ and $M_{k+1}$ are two sides of a common border. For circuit application, this condition can often be observed in systems whose switching is directly controlled by a comparator as shown in Fig. 4(a) (e.g. in the pulse-widthmodulation (PWM) controlled converter). This case is well covered by conventional modeling methods [Ma et al., 2004], and it clearly becomes a special case under the general modeling method proposed here. In some other systems, moreover, switchings may be controlled by a RS flip-flop or some other types of latches, e.g. in event-driven switching like the current-mode controlled converter [Fig. 4(b)]. Such systems have to be considered in the light of the new model formulation presented above. The new modeling approach is thus able to model a wider class of switched dynamical systems.

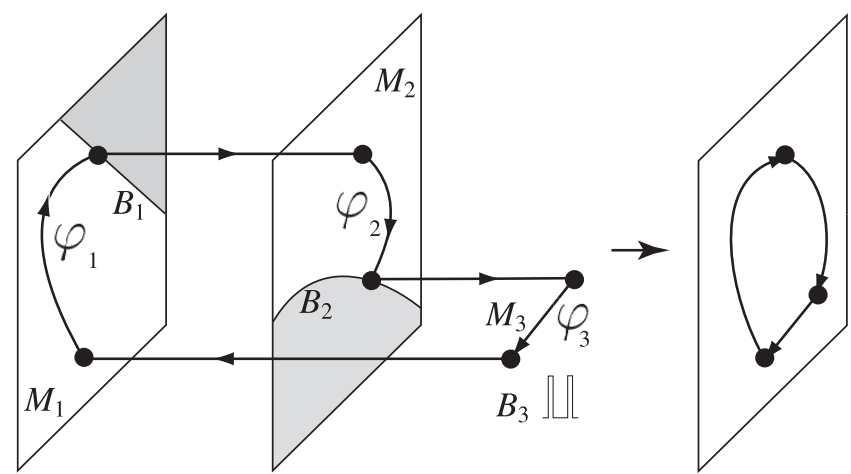

Fig. 3. Illustration of the proposed modeling method as applied to a system consisting of three subsystems.

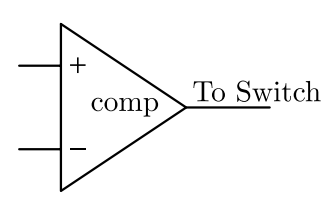

(a)

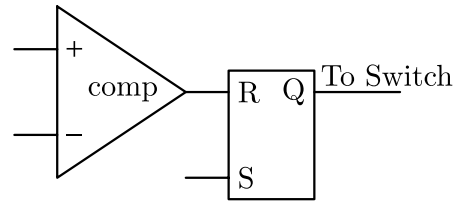

(b)
Fig. 4. Circuit implementations of common border. (a) PWM control leading to border crossing; (b) currentmode control leading to border reflecting. 


\section{Periodic Solution and First Return Map}

We first consider a general fundamental solution. Starting at $x_{0}$, a solution flow moves in $M_{1}$ at the very beginning, and then touches the border $B_{1}$ at $x_{1}$ after time $\tau_{1}$. Hitting the border causes a jump to $M_{2}$. Similarly, hitting border $B_{2} \in M_{2}$ will lead to a jump to $M_{3}$. In this way, the solution flow moving in $M_{k}$ will hit specific border $B_{k}$ and switch to $M_{k+1}$. Finally, it will get to $M_{m}$, the last space, and then return to $M_{1}$ at $x_{m}$ at time $\tau_{m}$. Thus, we can define a map as

$$
F_{m}: R^{n} \mapsto R^{n} ; \quad x_{0} \mapsto x_{m}
$$

which is similar to the definition of Poincaré map for autonomous systems. We simply refer to it as the first return map.

The flow of the fundamental solution described above can be constructed by following equations:

$$
\begin{aligned}
x_{1} & =\varphi_{1}\left(\tau_{1}, x_{0}\right) & & \left(x \in M_{1}\right) \\
& \vdots & & \\
x_{m} & =\varphi_{m}\left(\tau_{m}-\tau_{m-1}, x_{m-1}\right) & & \left(x \in M_{m}\right)
\end{aligned}
$$

Moreover, since the point where the flow hits the border satisfies the switching conditions (2), we have

$$
\begin{aligned}
& \beta_{1}\left(x_{1}, \tau_{1}\right)=0 \quad\left(\text { border } B_{1}\right) \\
& \beta_{m}\left(x_{m}, \tau_{m}\right)=0 \quad\left(\text { border } B_{m}\right)
\end{aligned}
$$

Because the flow is a fundamental periodic solution only if $x_{m}=x_{0}$, we can replace $x_{m}$ with $x_{0}$ to find the solution. Thus, from (6) and (7), we have $m \times n+m$ scalar equations. Meanwhile, the unknowns of (6) and (7) are $\left\{x_{0}, \ldots, x_{m-1}, \tau_{1}, \ldots, \tau_{m}\right\}$, and the total scalar number is $m \times n+m$. Thus, we can solve the periodic solution using an appropriate numerical method.

\section{Analysis of Stability and Bifurcation}

Stability of a periodic solution can be determined from the Jacobian of the first return map defined in (5), which is described by $\partial x_{m} / \partial x_{0}$. Since the flow is piecewise defined, we rewrite the Jacobian as

$$
\frac{\partial x_{m}}{\partial x_{0}}=\frac{\partial x_{m}}{\partial x_{m-1}} \frac{\partial x_{m-1}}{\partial x_{m-2}} \cdots \frac{\partial x_{2}}{\partial x_{1}} \frac{\partial x_{1}}{\partial x_{0}}=\prod_{i=1}^{m} \frac{\partial x_{i}}{\partial x_{i-1}} .
$$

From the $i$ th equation of (6), which can be written as $x_{i}=\varphi_{i}\left(\tau_{i}-\tau_{i-1}, x_{i-1}\right)$, we get

$$
\frac{\partial x_{i}}{\partial x_{i-1}}=\frac{\partial \varphi_{i}}{\partial t} \frac{\partial \tau_{i}}{\partial x_{i-1}}-\frac{\partial \varphi_{i}}{\partial t} \frac{\partial \tau_{i-1}}{\partial x_{i-1}}+\frac{\partial \varphi_{i}}{\partial x_{i-1}}
$$

where $\varphi_{i}$ and $\partial \varphi_{i} / \partial x_{i-1}$ denote $\varphi_{i}\left(\tau_{i}-\tau_{i-1}, x_{i-1}\right)$ and $\partial \varphi_{i} /\left.\partial x\right|_{x=x_{i-1}}$, respectively. Since $x_{i}$ and $x_{i-1}$ satisfy the switching conditions: $\beta_{i}\left(x_{i}, \tau_{i}\right)=0$ and $\beta_{i-1}\left(x_{i-1}, \tau_{i-1}\right)=0$, from which we can write

$$
\begin{aligned}
\frac{\partial \beta_{i}}{\partial x_{i-1}} & =\frac{\partial \beta_{i}}{\partial x} \frac{\partial x_{i}}{\partial x_{i-1}}+\frac{\partial \beta_{i}}{\partial t} \frac{\partial \tau_{i}}{\partial x_{i-1}}=0 \\
\frac{\partial \beta_{i-1}}{\partial x_{i-1}} & =\frac{\partial \beta_{i-1}}{\partial x}+\frac{\partial \beta_{i-1}}{\partial t} \frac{\partial \tau_{i-1}}{\partial x_{i-1}}=0 .
\end{aligned}
$$

Solving (10), we get $\partial \tau_{i} / \partial x_{i-1}$ and $\partial \tau_{i-1} / \partial x_{i-1}$. Putting them in (9) yields

$$
\frac{\partial x_{i}}{\partial x_{i-1}}=\frac{f_{i}\left(\varphi_{i}\right) \frac{\partial \beta_{i-1}}{\partial x}+\frac{\partial \varphi_{i}}{\partial x_{i-1}} \frac{\partial \beta_{i-1}}{\partial t}}{f_{i}\left(\varphi_{i}\right) \frac{\partial \beta_{i}}{\partial x}+\frac{\partial \beta_{i}}{\partial t}} \cdot \frac{\frac{\partial \beta_{i}}{\partial t}}{\frac{\partial \beta_{i-1}}{\partial t}}
$$

where $f_{i}\left(\varphi_{i}\right)$ represents $\partial \varphi_{i} / \partial t$. Note that $\partial \varphi_{i} / \partial x_{i-1}$ can be obtained by solving

$$
\frac{\mathrm{d}}{\mathrm{d} t}\left(\frac{\partial \varphi_{i}}{\partial x_{i-1}}\right)=\frac{\partial f_{i}}{\partial x}\left(\frac{\partial \varphi_{i}}{\partial x_{i-1}}\right),\left.\quad \frac{\partial \varphi_{i}}{\partial x_{i-1}}\right|_{t=0}=I_{n} .
$$

Thus, substituting (11) into (8) and using an appropriate numerical method, we can calculate the Jacobian of the first return map of a specific periodic solution. Finally, by finding the roots of the characteristic equation, we can determine the stability of the periodic solution.

\section{Illustrative Examples}

\subsection{Current-mode controlled boost converter}

Switched dynamical systems can be found in many practical applications. We will discuss the boost converter shown in Fig. 2. Since it may operate in continuous conduction mode and discontinuous conduction mode [Tse, 1994], the model can be represented schematically, as shown in Fig. 5.

We first consider the space $M_{1}$. The system with state $x=\left[v_{C}, i_{L}\right]^{T}$ is described by

$$
S_{1}: \dot{x}=\left[\begin{array}{cc}
-\frac{1}{R C} & 0 \\
0 & 0
\end{array}\right] x+\left[\begin{array}{c}
0 \\
\frac{1}{L}
\end{array}\right] E .
$$




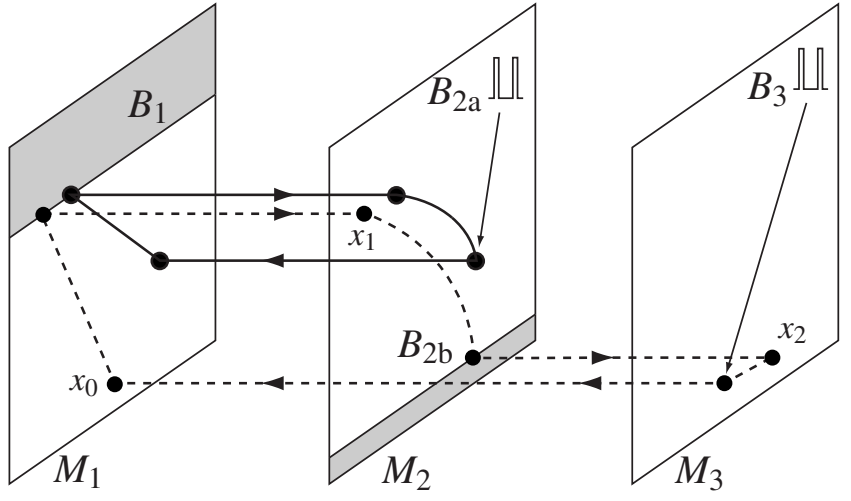

Fig. 5. Model of current-mode controlled boost converter with fixed current reference.

In $M_{1}$, there is a border $B_{1}$, which is defined by

$$
B_{1}=\left\{(x, t) \in R^{2} \times R: \beta_{1}=x(2)-I_{\text {ref }}=0\right\} .
$$

When $x$ hits $B_{1}$, switching occurs and $x$ jumps to $M_{2}$, where the system becomes

$$
S_{2}: \dot{x}=\left[\begin{array}{cc}
-\frac{1}{R C} & \frac{1}{C} \\
-\frac{1}{L} & 0
\end{array}\right] x+\left[\begin{array}{c}
0 \\
\frac{1}{L}
\end{array}\right] E .
$$

Two borders are available in $M_{2}$. One is the clock signal for resetting the switch, the other is observed when the inductor current drops to zero. We can write the border function as

$$
\begin{aligned}
& B_{2 a}=\left\{(x, t) \in R^{2} \times R: \beta_{2 a}=t-k T=0\right\}, \\
& B_{2 b}=\left\{(x, t) \in R^{2} \times R: \beta_{2 b}=x(2)=0\right\} .
\end{aligned}
$$

Once $x$ touches $B_{2 a}$, it will return to $M_{1}$ (as indicated by the solid line). Moreover, if it reaches $B_{2 b}$ ahead of $B_{2 a}$, it jumps to $M_{3}$. The dynamics in $M_{3}$ is described simply by

$$
S_{3}: \dot{x}=\left[\begin{array}{cc}
-\frac{1}{R C} & 0 \\
0 & 0
\end{array}\right] x .
$$

When the clock signal arrives, i.e. when $x$ hits $B_{3}$,

$$
B_{3}=\left\{(x, t) \in R^{2} \times R: \beta_{3}=t-k T=0\right\},
$$

the state will return to $M_{1}$ (as shown by the dashed line). Thus any solution of the current-mode controlled boost converter can be solved and its stability can be analyzed by the method introduced in Sec. 2.
We consider the simplest case of the period-1 solution, which is the preferred operation in practice. Basically, the solution under the so-called continuous conduction mode can be constructed as

$$
\begin{aligned}
x_{1} & =\varphi_{1}\left(\tau_{1}, x_{0}\right), \\
x_{0} & =\varphi_{2}\left(\tau_{2}-\tau_{1}, x_{1}\right), \\
x_{1}(2)-I_{\text {ref }} & =0, \\
\tau_{2}-T & =0 .
\end{aligned}
$$

Thus, five scalar unknowns $x_{1}, x_{2}, \tau_{1}$ can be obtained. Moreover, by fixing some parameters in Fig. 2 as

$$
L=1.5 \mathrm{mH}, \quad T=100 \mu \mathrm{s}, \quad I_{\mathrm{ref}}=0.8 \mathrm{~A},
$$

we get the bifurcation diagram shown in Fig. 6 . Three bifurcation curves are given corresponding to different capacitor values. Stable period-1 solutions can be observed on the left-hand side of these curves. As we move across the bifurcation curves from left to right, the solution becomes period-2 and exhibit more complicated behavior, such as border collision and chaos.

\subsection{Current-mode controlled boost converter with voltage feedback}

In practice, the aforementioned current-mode boost converter is almost always equipped with a

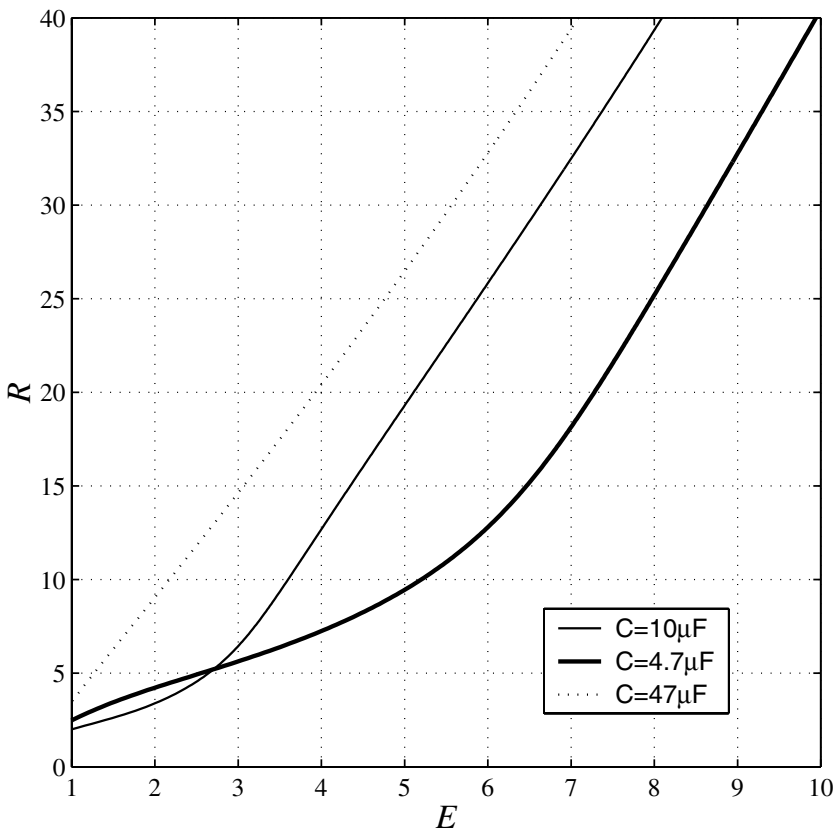

Fig. 6. Bifurcation diagram in $(E, R)$ plane of current-mode controlled boost converter with fixed current reference. 
voltage feedback loop that regulates the output voltage level [Tse, 2003]. Essentially, as shown in Fig. 7, we add a proportional-integral control feedback unit to the same boost converter studied in the previous subsection. Thus, $i_{\text {ref }}$ is no longer a constant; it becomes a state variable, and the system is 3-dimensional. The condition for turning off the switch is $i_{L}=i_{\text {ref }}$, which is a fixed plane in the three-dimensional space. A schematic diagram of the system model is shown in Fig. 8.

In the feedback control system, additional parameters related to the feedback loop are introduced. With the notations of Fig. 8, we assume some terms as following

$$
\tau_{f}=R_{f} C_{f}, \quad G_{f}=\frac{R_{f}}{R_{1}}, \quad I_{\mathrm{ref}}=\frac{R_{f}+R_{2}}{R_{2}} V_{\mathrm{ref}},
$$

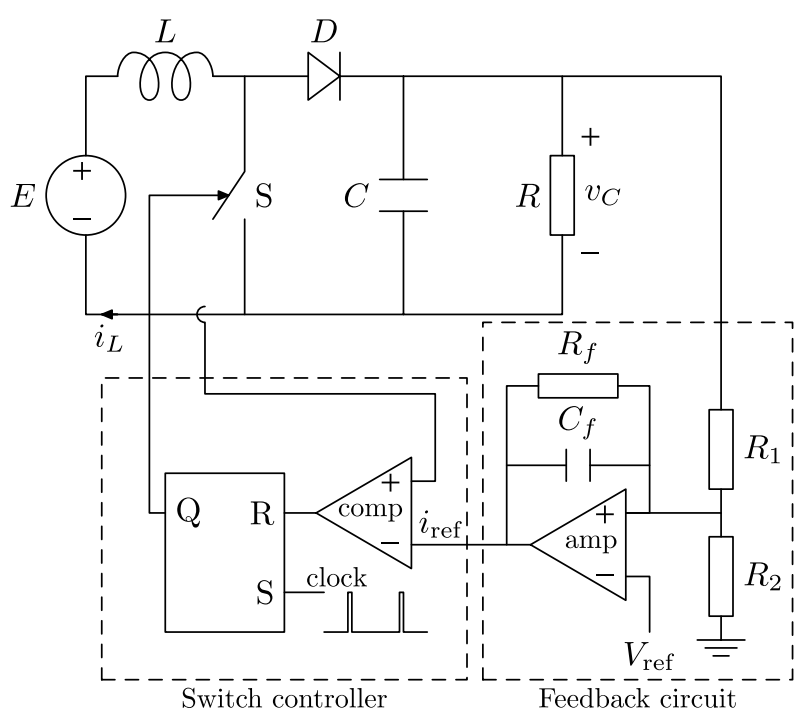

Fig. 7. Current-mode controlled boost converter with proportional-integral voltage feedback loop.

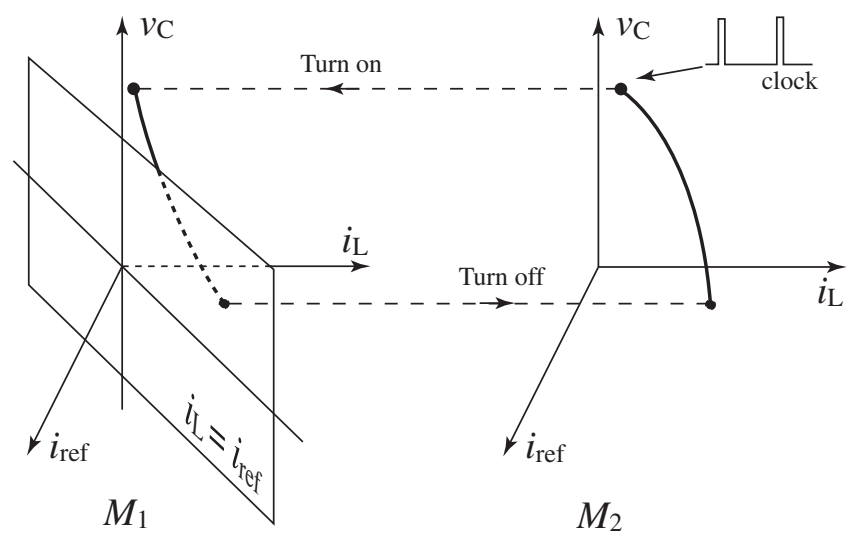

Fig. 8. System model of current-mode controlled boost converter with voltage feedback loop.
In space $M_{1}$, the switch is turned on. With the state variable $x=\left[v_{C}, i_{L}, i_{\text {ref }}\right]^{T}$, we write the system equation as

$$
S_{1}: \dot{x}=\left[\begin{array}{ccc}
-\frac{1}{R C} & 0 & 0 \\
0 & 0 & 0 \\
-\frac{G_{f}}{\tau_{f}} & 0 & -\frac{1}{\tau_{f}}
\end{array}\right] x+\left[\begin{array}{c}
0 \\
\frac{E}{L} \\
\frac{I_{\mathrm{ref}}+G_{f} V_{\mathrm{ref}}}{\tau_{f}}
\end{array}\right] .
$$

The border in space $M_{1}$ is a fixed plane defined by

$$
B_{1}=\left\{(x, t) \in R^{3} \times R: \beta_{1}=x(2)-x(3)=0\right\} .
$$

Hitting the above plane, the solution jumps to $M_{2}$, where the system equation is defined by

$$
S_{2}: \dot{x}=\left[\begin{array}{ccc}
-\frac{1}{R C} & \frac{1}{C} & 0 \\
-\frac{1}{L} & 0 & 0 \\
-\frac{G_{f}}{\tau_{f}} & 0 & -\frac{1}{\tau_{f}}
\end{array}\right] x+\left[\begin{array}{c}
0 \\
\frac{E}{L} \\
\frac{I_{\mathrm{ref}}+G_{f} V_{\mathrm{ref}}}{\tau_{f}}
\end{array}\right] \text {. }
$$

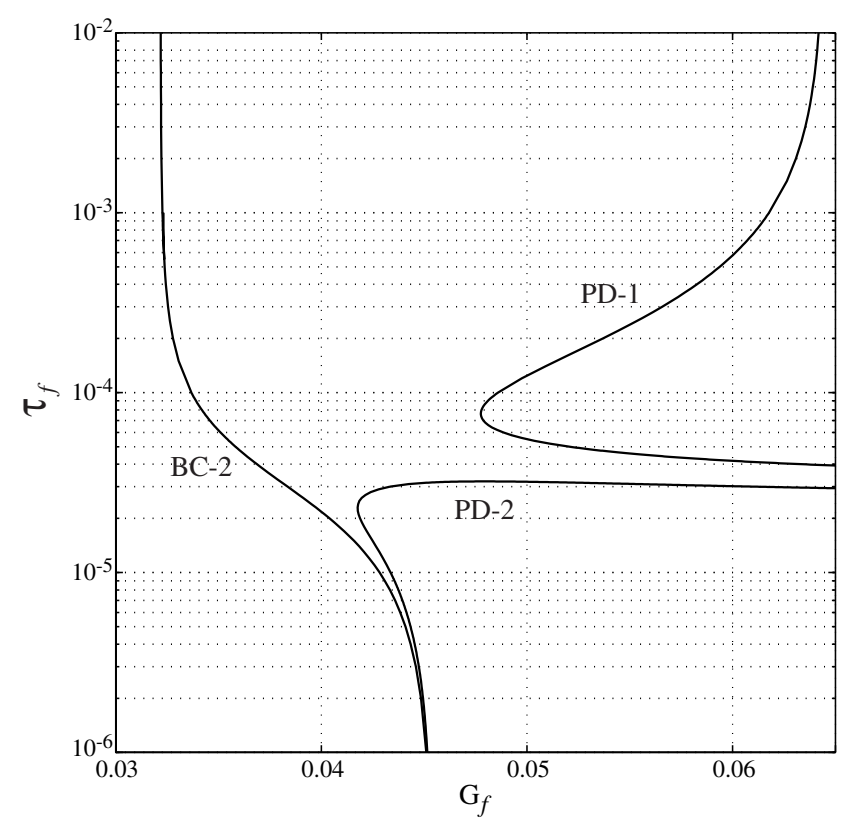

Fig. 9. Bifurcation diagram in $\left(G_{f}, \tau_{f}\right)$ plane for proportional-integral voltage feedback current-mode controlled boost converter. 
In space $M_{2}$, border is defined by periodic clock signal.

$$
B_{2}=\left\{(x, t) \in R^{3} \times R: \beta_{2}=t-k T=0\right\} .
$$

When the clock signal arrives, the switch is turned on, and the solution jumps back to $M_{1}$.

Using the method described in Sec. 2, we can solve the periodic solution and investigate the bifurcation problem of the above voltage feedback current-mode controlled boost converter. Some parameters are chosen as follows:

$$
\begin{gathered}
L=1.5 \mathrm{mH}, \quad E=10 \mathrm{~V}, \quad R=40 \Omega, \\
T=100 \mu \mathrm{s}, \quad \tau_{C}=\frac{C R}{T}=2, \\
R_{f}=10 \mathrm{k} \Omega, \quad R_{2}=50 \mathrm{k} \Omega, \quad V_{\text {ref }}=1.5 \mathrm{~V} .
\end{gathered}
$$

A bifurcation diagram in the $\left(G_{f}, \tau_{f}\right)$ plane can be generated numerically, as shown in Fig. 9. In the bifurcation diagram, abbreviations $\mathrm{PD}$ and $\mathrm{BC}$ denote period-doubling bifurcation and border collision, respectively. The region to the right of bifurcation curve PD-1 corresponds to period-1 solution, as shown in Fig. 10(a). Crossing PD-1 from right to left, period-doubling occurs and a period-2 solution such as the one shown in Fig. 10(b) can be observed. If the values of parameters cross the curve denoted by $\mathrm{BC}-2$, border collision occurs and a period- 4 solution with one skipped cycle (see Fig. 10(c)) comes into existence. A period-doubling bifurcation, which leads to a normal period- 4 solution, is also available for the period-2 solution. (Switching converters described by three-dimensional ODE were seldom considered. The bifurcation
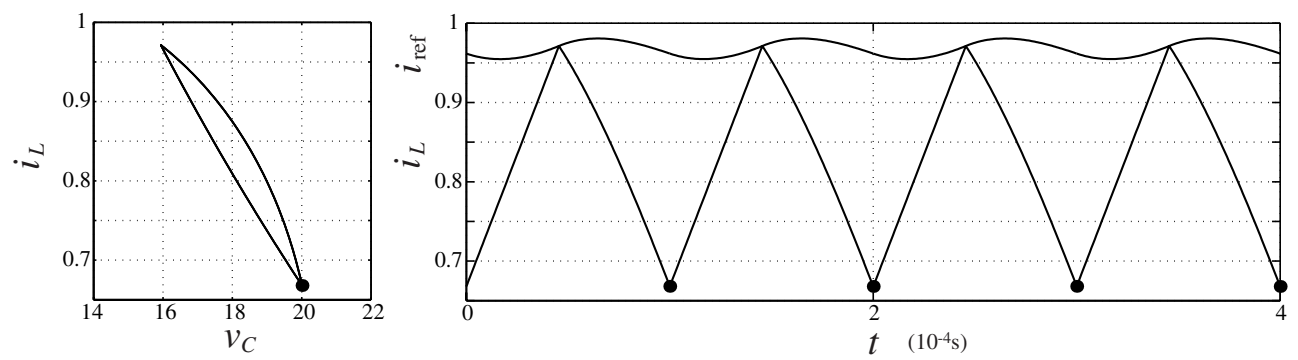

(a) $\left(G_{f}, \tau_{f}\right)=\left(0.06,10^{-4}\right)$
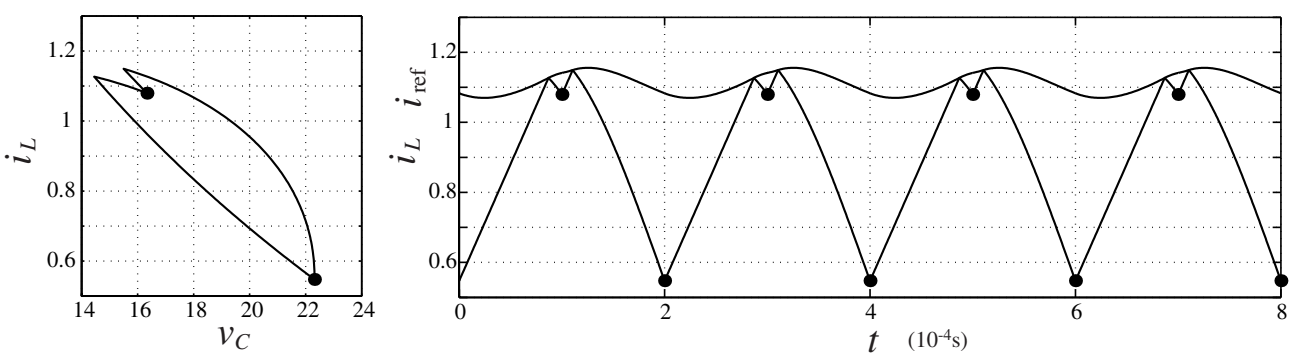

(b) $\left(G_{f}, \tau_{f}\right)=\left(0.04,10^{-4}\right)$
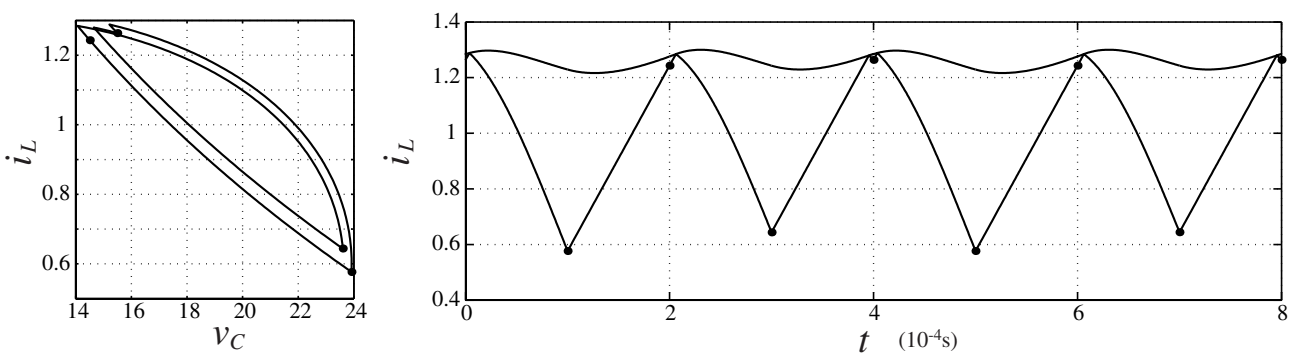

(c) $\left(G_{f}, \tau_{f}\right)=\left(0.03,10^{-4}\right)$

Fig. 10. Waveforms and phase portraits of (a) period-1 solution, (b) period-2 solution and (c) period-4 solution. 
diagram of such systems is obtained here for the first time.)

\section{Conclusion}

In this paper, we propose a general framework for modeling and analyzing switched dynamical systems. The method is capable of modeling most switched systems. Specifically, in the proposed modeling method, the switching dynamics is examined under a different operational viewpoint. First, subsystems and their borders are defined in separate spaces, allowing the systems to be modeled without a common border, thus covering a much wider class of possible switching scenarios. Second, the method is based on tracking down the solution flow, instead of solving the solution. The boost converter circuits have been used as examples for illustration. In principle, any piecewise linear/nonlinear models can be analyzed by this general method.

\section{Acknowledgment}

This work was supported in part by the Research Grant Council of Hong Kong under a competitive-bid earmarked research grant (No. PolyU 5241/03E).

\section{References}

Banerjee, S., Ranjan, P. \& Grebogi, C. [2000] "Bifurcation in two-dimensional piecewise smooth maps - theory and applications in switching circuits," IEEE Trans. Circuits Syst.-I 47, 633-643.

Banerjee, S. \& Verghese, G. [2001] Nonlinear Phenomena in Power Electronics: Attractors, Bifurcations, Chaos, and Nonlinear Control (IEEE Press, NY).

Chan, W. C. Y. \& Tse, C. K. [1997] "Study of bifurcations in current-programmed dc/dc boost converters: From quasi-periodicity to period-doubling," IEEE Trans. Circuits Syst.-I 44, 1129-1142.

Kousaka, T., Ueta, T. \& Kawakami, H. [1999] "Bifurcation of switched nonlinear dynamical systems," IEEE Trans. Circuits Syst.-II 46, 878-885.

Kousaka, T., Ueta, T. \& Kawakami, H. [2001] "Chaos and control of periodically switched nonlinear systems," Latin American Appl. Res. 31, 211-218.

Krupar, J., Mögel, A. \& Schwarz, W. [2004] "Continuous-discrete systems - modelling and statistical analysis," Proc. Int. Symp. Nonlinear Theory \& Its Applications, Vol. 1, pp. 55-58.

Ma, Y., Kawakami, H. \& Tse, C. K. [2004] "Analysis of bifurcation in switched dynamical systems with periodically moving borders," IEEE Trans. Circuits Syst.-I 51, 1184-1193.

Tse, C. K. [1994] "Flip bifurcation and chaos in threestate boost switching regulators," IEEE Trans. Circuits Syst.-I 41, 16-23.

Tse, C. K. [2003] Complex Behavior of Switching Power Converters (CRC Press, Boca Raton). 